
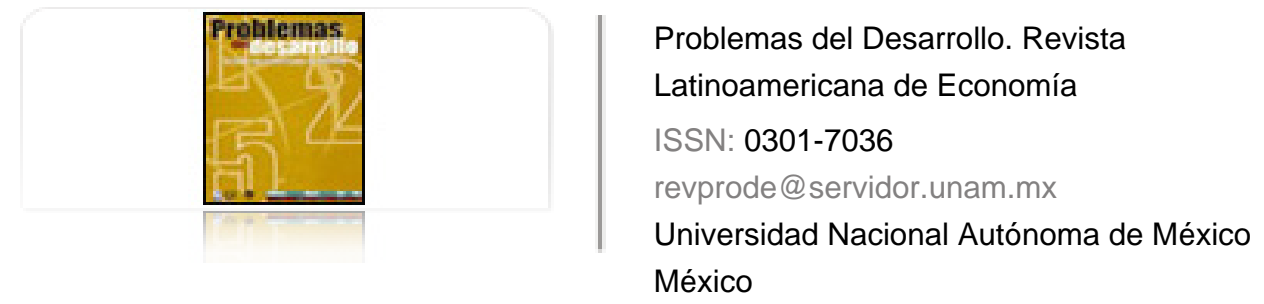

FUENTES, NOÉ ARÓN; SASTRÉ GUTIÉRREZ, MYRNA

Evaluación de la congruencia entre economía y gobierno en torno al desarrollo regional de Baja California Sur, México

Problemas del Desarrollo. Revista Latinoamericana de Economía, vol. 32, núm. 126, abril-julio, 2001, pp. $149-174$

Universidad Nacional Autónoma de México

Distrito Federal, México

Disponible en: http://www.redalyc.org/articulo.oa?id=11820094007

Cómo citar el artículo

- Número completo

- Más información del artículo

Página de la revista en redalyc.org

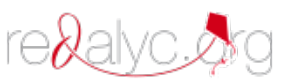

Sistema de Información Científica

Red de Revistas Científicas de América Latina, el Caribe, España y Portugal Proyecto académico sin fines de lucro, desarrollado bajo la iniciativa de acceso abierto 


\title{
EVALUACIÓN DE LA CONGRUENCIA ENTRE ECONOMÍA Y GOBIERNO EN TORNO AL DESARROLLO REGIONAL de Baja California Sur, MÉXICO
}

\author{
NOÉ ARÓN FUENTES* \\ MYRNA SASTRÉ GUTIÉRREZ**
}

\begin{abstract}
RESUMEN
En el estado mexicano de Baja California Sur (BCS) se está llevando a cabo una discusión sobre la mejor estrategia de desarrollo económico regional, que le permita al estado aprovechar sus potencialidades y desarrollar algunas nuevas con una estrategia definida en el corto y en el largo plazos. En la discusión de esta estrategia, el concepto de sector clave está jugando un papel fundamental, aunque éste se ha entendido de diversas maneras. El hecho de conceptualizar de manera diferente el término de sector clave no contribuye en la tarea de diseñar una estrategia de desarrollo que sea consistente en el corto y en el largo plazos, sobre todo cuando se busca que estos sectores sean los ejes de la misma. En este trabajo presentamos un análisis relacionado con la identificación de sectores clave para el estado de BCS, mediante el auxilio de varias metodologías que tienen como base el sistema de insumo-producto (MIP).

Palabras clave: Baja California Sur, sectores clave, matriz de insumo-producto.
\end{abstract}

\footnotetext{
* Director del Departamento de Estudios Económicos de El Colegio de la Frontera Norte. Correo electrónico: <afuentes@colef.mx>.

** Estudiante de la Maestría en Economía Aplicada, Promoción 1998-2000.
} 


\section{Evaluation of the Congruence Between the Economy and Government as Regards the REgional Development of Baja California Sur, Mexico ABSTRACT}

The mexican state of Baja California Sur (BCS) is currently engaged in a discussion on the best strategy for regional economic development that will enable the state to take advantage of its potentialities while developing new ones with a well-defined strategy in both the long and short term. In the discussion of this strategy, the concept of key sector plays a key role, although it has been understood in a variety of ways. The fact of conceptualizing the term key sector differently has not helped the task of designing a development strategy that will be consistent in both the long and short term, particularly when the aim is for these sectors to serve as axes of the latter. This paper presents an analysis linked to the identification of key sectors for the state of BCS, using various types of methodology based on the input-product matrix (IMP).

Key products: Baja California Sur, key sectors, input-product matrix.

$$
\begin{gathered}
\text { Évaluation DE la COHÉRENCE ENTRE ÉCONOMIE } \\
\text { ET GOUVERNEMENT DANS LE DÉVELOPPEMENT RÉGIONAL } \\
\text { DE L'ÉTAT DE BAJA CALIFORNIA SUR, MEXIQUE } \\
\text { RÉSUMÉ }
\end{gathered}
$$

Dans l'État de Baja California Sur (BCS), l'on discute actuellement sur la meilleure stratégie de développement économique régional à adopter pour que l'État soit à même de tirer parti de ses potentialités, et d'en développer de nouvelles, dans le cadre d'une stratégie à court et à long terme. Dans ces débats, l'idée de secteur-clé, bien qu'entendue de manières diverses, tient une place fondamentale. Les conceptions diverses du terme secteur-clé ne facilitent pas l'élaboration d'une stratégie de développement cohérente à court et à long terme, surtout si l'on veut que ces secteurs en constituent le moteur. L'auteur de ces lignes présente une analyse liée au ciblage de secteurs-clés pour l'État de BCS à l'aide de plusieurs méthodologies se basant sur le système d'entréessorties (MIP).

Mots-clés : Baja California Sur, secteurs-clés, matrice d'entrées-sorties. 


\section{INTRODUCCIÓN}

$\mathrm{E}$

n el estado mexicano de Baja California Sur (BCS) se está llevando a cabo una discusión sobre la mejor estrategia de desarrollo económico regional, que le permita aprovechar sus potencialidades y desarrollar algunas nuevas con una estrategia definida en el corto y largo plazos. ${ }^{1}$

En la discusión de esta estrategia, el concepto de sector clave está desempeñando un papel fundamental, aunque se ha entendido de diversas maneras. Primero, dicha conceptualización clasifica los sectores clave de manera muy general, como actividades económicas: primarias, secundarias y terciarias. ${ }^{2}$ Segundo, define a los sectores como complejos industriales, caracterizados por ser grupos de actividades industriales que presentan un fuerte dinamismo y con relaciones iterativas, es decir, relaciones en un solo sentido. ${ }^{3}$ Tercero, determina a los sectores como polarizados o enclaves, caracterizados por ser grupos de actividades interrelacionadas de manera circular, es decir, mutuas entre dos ramas. ${ }^{4}$ Cuarto, fija a los sectores como líderes, caracterizados por ser grupos de actividad que presentan un fuerte dinamismo, pero escasa relación con el resto de la economía (no se incluye ningún elemento de ponderación tal como el empleo, valor agregado, etc.). ${ }^{5} \mathrm{El}$ hecho de conceptualizar de manera diferente el término de sector clave no contribuye en la tarea de diseñar una estrategia de desarrollo que sea consistente en el corto plazo, sobre todo cuando se busca que estos sectores sean los ejes de la misma.

En este trabajo presentamos un análisis relacionado con la identificación de sectores clave para el estado de Baja California Sur (BCS), México, mediante el auxilio de varias metodologías que tienen como base el sistema de insumoproducto (MIP).

Los trabajos de N. P. Rasmussen (1956), A. O. Hirschman (1958), H.B. Chenery y T. Watanabe (1958) y B.R. Hazari (1970), suministran las metodologías alternativas para el estudio empírico de los sectores clave. Específicamente, éstos se definen así: $a$ ) de acuerdo con sus encadenamientos hacia atrás y hacia delante, a la manera de Chenery y Watanabe (1958); $b$ ) en términos puramente tecnológicos, a la manera de Rasmussen (1956) y Hirschman (1958), y c) de

1 BCS está reorientando su política económica debido a dos hechos trascendentes: 1) la pérdida de la ventaja comparativa que le brindaba el ser Zona Libre; y, 2) la disminución de los subsidios federales.

2 Véase Arthur Andersen \& Co. A.C.-Gobierno del estado de Baja California Sur, Programa Estratégico del Desarrollo Económico para el estado de BCS, 4 vols., La Paz, BCs, México, 1992.

3 Véase Plan Estatal de Desarrollo 1987-1993, BCs.

4 Véase "Estrategia de desarrollo", Plan Estatal de Desarrollo 1993-1999, Parte II, BCS.

5 Véase Plan Estatal de Desarrollo 1992-2005, BCS. 
acuerdo a una función de preferencia - $\mathrm{u}$ objetivo - de los tomadores de decisiones políticas dada la estructura tecnológica, a la manera de Hazari (1970).

Los índices de Rasmussen, Hirschman, Chenery y Watanabe han sido ampliamente utilizados en estudios regionales para la selección de sectores relevantes en los procesos de crecimiento económico y de cambio estructural. Es decir, y como una primera aproximación, sirven para la identificación de grupos de actividades industriales fuertemente interrelacionadas, entendiendo por tales, aquellos grupos de actividades con índices superiores a la media.

Asimismo, se ha señalado su utilidad para expresar la coherencia a corto plazo de determinadas decisiones de política económica regional, porque se podría considerar a las industrias seleccionadas como las más relevantes para la consecución de determinadas tasas de crecimiento económico regional. De hecho, y según los autores anteriormente citados, se debería incentivar a estos sectores clave para maximizar la tasa de crecimiento económico de una región 0 área. ${ }^{6}$

Analizada bajo estas dos perspectivas - como detonadores de desarrollo económico y como instrumentos de política económica-, la identificación de sectores clave puede constituirse en determinante para el desarrollo económico regional.

Así, pues, la pertinencia del estudio puede justificarse basada por tres consideraciones:

1. La inexistencia de un análisis en el ámbito oficial que identifique las interrelaciones productivas clave en el proceso de desarrollo económico del estado de BCS.

2. La importancia de que en este análisis se considere el peso de los objetivos de política pública, puesto que en la economía estatal parecen predominar los sectores independientes (es decir, enclave o polarizantes).

3. La posibilidad de poder distinguir empíricamente el concepto de sector clave del de sector líder. ${ }^{7}$

El trabajo se organiza de la siguiente manera. En la primera sección se expone el concepto de encadenamiento interindustrial (linkage); en la segunda se

6 Como se sabe, Hirschman (1958) introduce y Porter (1990) expande el tema de los encadenamientos en el estudio del desarrollo económico; en esencia su hipótesis señala que: "si se incentivan los sectores más encadenados de una economía, a largo plazo se conseguirán mayores tasas de crecimiento económico".

7 Conceptualmente, y de acuerdo con Oosterhaven (1981), sectores líderes son aquellos que tienen un impacto positivo y muy grande en el desarrollo de sus respectivas regiones, es decir, que son capaces de liderar el desarrollo de una región. Asimismo, y de acuerdo al mismo J. Oosterhaven (1981) y a Hewings (1982), sectores clave son aquellos que mediante la fuerza de sus encadenamientos intersectoriales podrían probablemente ejercer una influencia significativa en los niveles de producción de otros sectores y cosecuentemente en el empleo regional, valor agregado y otras variables objetivo. 
analizan los coeficientes de Chenery y Watanabe; en la tercera se analizan los coeficientes de Rasmussen, que permiten medir el poder de dispersión y la sensibilidad de dispersión de cada sector o industria; en la cuarta sección se incorporan ponderaciones a los coeficientes de Rasmussen con la inclusión en el análisis de una función objetivo del planeador o tomador de decisiones, y en la quinta se presenta la evidencia empírica sobre los sectores clave del estado de BCS. Finalmente, se presentan conclusiones.

\section{EL CONCEPTO DE ENCADENAMIENTO}

El modelo estático abierto de Leontief o MIP, al cuantificar las relaciones entre las diversas ramas de actividad como oferentes o demandantes mutuas de insumos intermedios, permite seleccionar industrias clave en función de la importancia de las interdependencias que pueden cuantificarse por medio de los encadenamientos interindustriales. La idea central de este tipo de estudios es que no todas las actividades económicas tienen igual capacidad para inducir efectos sobre otras, ya que algunas se caracterizan por provocar efectos de arrastre o de empuje más intensos, previéndose una posible correlación entre estos efectos y los niveles de desarrollo económico.

Hirschman (1958) distinguió entre encadenamientos hacia atrás (backward linkages), medidos por la capacidad de una actividad para provocar el desarrollo de otras cuando utiliza insumos procedentes de éstas, y encadenamientos hacia adelante (forward linkages), que se producen cuando se desarrolla una actividad que obtiene productos que utilizarán otras ramas posteriores como insumos intermedios para su proceso de producción.

\section{Los COEFICIENTES DE CHENERY y WaTANABE}

Chenery y Watanabe (1958) efectuaron una cuantificación de los encadenamientos, con la selección de aquellas actividades cuyos efectos de encadenamiento eran superiores a la media. De esta forma realizaron una clasificación cuatripartita de actividades en función de la combinación de dos criterios, a saber: $a$ ) utilización por parte de cada rama de insumos intermedios con respecto a su producción, y $b$ ) destino intermedio de los productos de cada rama con respecto al total de los destinos. ${ }^{8}$

8 En particular estos autores definen los índices $\mu_{\mathrm{i}} \mathrm{y} \omega_{\mathrm{i}}$ de la forma siguiente:

$$
\begin{array}{cc}
\mu_{i}=\sum_{i} x_{i j} / X_{j} & \omega_{j}=\sum x_{i j} / Z_{i} \\
j
\end{array}
$$

Donde $X$, y $Z$ son, respectivamente, la producción efectiva de la rama $j$ y el producto total de la $i$. Asimismo, $x_{i j}$ es la utilización que la rama $j$ hace de insumos intermedios de la rama $i$. 
Así, las actividades industriales se agrupan en cuatro tipos: I) actividades con fuertes encadenamientos hacia adelante y hacia atrás (sectores clave); II) actividades con altos encadenamientos hacia delante y bajos hacia atrás (sectores base); III) actividades con bajos encadenamientos hacia delante y altos hacia atrás (sectores con fuerte arrastre); y IV) actividades con bajos encadenamientos hacia atrás y hacia delante (sectores independientes). Las ramas se ordenan al comparar sus coeficientes con la media de todas las ramas. Véase esquema 1.

ESQUEMA 1

$\omega_{\mathrm{i}}>\bar{\omega}_{\mathrm{i}}$

$\omega_{\mathrm{j}}<\bar{\omega}_{\mathrm{j}}$

\begin{tabular}{|c|c|c|}
\hline$\mu_{\mathrm{i}}<\bar{\mu}_{\mathrm{i}}$ & $\begin{array}{l}\text { III. No manufacturas } \\
\text { (destino intermedio) }\end{array}$ & $\begin{array}{l}\text { IV. No manufacturas } \\
\text { (destino final) }\end{array}$ \\
\hline$u_{j}>$ & $\begin{array}{l}\text { I. Manufacturas } \\
\text { (destino intermedio) }\end{array}$ & $\begin{array}{l}\text { II. Manufacturas } \\
\text { (destino final) }\end{array}$ \\
\hline
\end{tabular}

El orden de las actividades señalado en el párrafo anterior es relevante, puesto que las actividades con fuertes encadenamientos hacia atrás y hacia adelante (actividades manufactureras de destino intermedio) a la vez serán clave para provocar sobre ellas presiones de desarrollo por su mayor capacidad para estimular a otras actividades económicas. La ordenación de los grupos siguientes se justifica porque encadenamientos hacia atrás se consideran más importantes que los encadenamientos hacia adelante 0 , al menos, su potencialidad para ser creados una vez que aparece una industria nueva o más verosímil.

El análisis de Chenery y Watanabe, sin embargo, presenta algunas limitaciones importantes:

a) En el análisis se utilizan los coeficientes directos de la MIP, ya que según los autores facilitan la comparación entre ramas porque aislan la fuente de las diferencias. Esta observación es correcta si se agrega directas, ya que aunque las diferencias indirectas no se podrían asignar rama por rama, sí pueden cuantificarse mediante el uso de la matriz inversa, y esto suministra una información más útil, y más genuina de una MIP, que la exclusiva cuantificación de los efectos directos.

b) Igualmente, puede señalarse que los coeficientes obtenidos representan medidas sin consideración alguna sobre las desviaciones, por lo que no se distingue entre las vinculaciones muy concentradas en pocas ramas y las 
muy difundidas, lo que no es irrelevante desde el punto de vista del desarrollo económico.

c) Por último, los índices de Chenery-Watanabe no son ponderados, es decir, no tienen en cuenta la relativa capacidad de cada actividad para estimular el desarrollo en diferente magnitud. ${ }^{9}$

No hay que dejar de destacar que para Chenery-Watanabe sus índices son más útiles para señalar el carácter general de la interdependencia que para un análisis de sectores específicos, lo cual es relevante como precaución inmediata de utilizarlos con pretensiones de incidencia inmediata en la política económica. ${ }^{10}$

\section{LOS ÍNDICES DE RASMUSSEN}

Los índices que se obtienen según la versión Chenery-Watanabe han sido perfeccionados incorporando tres elementos: 1) la realización de operaciones con los coeficientes de la inversa de Leontief; 2) la incorporación de ponderaciones, y 3) la medición de la dispersión de los efectos.

Rasmussen (1956) incorpora estos elementos, compara el grado de interdependencia de una industria con todas y deja definidos los índices en forma tal que sólo han experimentado, con posterioridad, revisiones menores.

El autor utilizó los coeficientes de la inversa de la MIP de Leontief con objeto de calcular los efectos totales de una industria sobre las demás -y no sólo los efectos directos. Sumando las columnas de la MIP inversa obtuvo el poder de dispersión de una industria o la expansión de sus efectos sobre el sistema industrial (expansión ocasionada en el sistema industrial al incrementar la demanda en una unidad para la industria $j$ ).

Este coeficiente se define como $U_{j}$.

$$
U_{j}=\frac{\frac{1}{n} Z_{i}}{\frac{1}{n^{2}} \sum_{i=1}^{n} Z \bullet{ }_{j}}
$$

9 Estas dificultades hicieron que los autores, que participaron en una polémica sobre la evidencia empírica de la hipótesis del crecimiento desequilibrado, se decantaran con sus índices y optaran por la utilización de los índices de Rasmussen.

10 Un comentario final. No son simétricos los coeficientes $\mu_{\mathrm{i}} \mathrm{y} \omega_{\mathrm{i}}$, los $\mu_{\mathrm{i}}$ no incluyen las importaciones en el denominador (por eliminar un importante factor de distorsión de los coeficientes al efectuar comparaciones entre países), mientras que las exportaciones sí se incluyen en los coeficientes $\omega_{\mathrm{i}}$. 
Siendo $n$ el número de ramas de actividad y

$$
Z \bullet{ }_{j}=\sum_{i=1}^{n} Z_{i j}
$$

siendo $z_{i j}$ el elemento genérico de la inversa de Leontief. ${ }^{11}$

En el numerador se reflejan los usos medios que una rama $(j)$ hace de la producción de otras. ${ }^{12} \mathrm{Al}$ comparar los coeficientes de uso con el medio de todas las ramas (denominador) se obtienen ramas con más poder de dispersión que la media $\left(U_{j}>1\right)$, que son ramas con gran capacidad de arrastre sobre otras. Cuando aumenta la demanda final de las mismas, arrastran a otras más intensamente que la media.

De la misma forma se definen los índices de sensibilidad de dispersión de $i$ (Ui) que expresan cómo la rama $i$ es arrastrada cuando aumenta en una unidad la demanda final en todas las ramas o la medida en que $i$ es arrastrada (demanda del producto de $j$ ) por la expansión en el sistema industrial. Es decir,

$$
U_{i}=\frac{\frac{1}{n} Z_{i} \bullet}{\frac{1}{n^{2}} \sum_{i} Z_{i}}
$$

Siendo $Z_{i}=\sum_{i=1}^{n} Z_{i j}$, e interpretándose el resto de los elementos como en el caso anterior.

\section{Incorporación de las ponderaciones}

Estos índices son susceptibles de perfeccionamiento en la medida en que industrias con índices iguales pueden no afectar de igual forma (o ser afectadas) al resto del sistema debido a que: $a$ ) no todas las industrias tienen el mismo peso en el sistema (midiéndolo, por ejemplo, por el peso de cada rama en la de-

11 El denominador, más claramente, es:

$$
\frac{1}{n^{2}} \sum_{j} \sum_{i} Z \bullet j=\frac{1}{n^{2}} \sum_{j} Z \bullet j=\frac{1}{n^{2}} \sum_{i} Z_{i}
$$

12 "El crecimiento directo o indirecto en el producto que ha de ser ofrecido por una industria elegida al azar si la demanda final para los productos de una industria (j) crece en una unidad" (Rasmussen, 1958: 133). 
manda final), y $b$ ) una industria puede tener un alto índice de dispersión pero muy concentrado en un reducido número de industrias.

El primer problema es el de las ponderaciones. Esta cuestión es importante, puesto que un incremento en la demanda final no se distribuye uniformemente en todas las industrias. Si se considera que ese incremento se distribuye entre todas las industrias en función de su participación en la demanda final igual a $n$ significaría para la industria $i$ :

$$
\frac{n X_{i \Delta}}{\sum_{i=1}^{n} X^{n \Delta}}
$$

Incorporando esta ponderación en la suma de los elementos de cada fila se tendría para la rama $j$ :

$$
Z_{i}^{w} \cdot \frac{\mathrm{n} \sum_{j=1}^{n} Z_{i j} X_{j \Delta}}{\left[\sum_{j=1}^{n} X_{j \Delta}\right]}
$$

Por lo que los incrementos se distribuyen entre las industrias en función de su participación en la demanda final. Definiendo la media total:

$$
\frac{1}{n^{2}} \sum_{i} Z_{i}^{w}
$$

Se tiene el índice ponderado de sensibilidad de dispersión:

$$
U_{\Delta}^{w}=\frac{\frac{1}{n} Z_{j}^{w}}{\frac{1}{n^{2}} \sum_{i} Z_{i}^{w}}
$$

Índice similar al obtenido con anterioridad pero que pondera las interrelaciones por el peso de las diferentes ramas en la demanda final.

\section{El problema de la dispersión de los efectos}

Los índices calculados hasta ahora son promedios y, por ello, sensibles a los valores extremos. Los efectos pueden estar concentrados en muy pocas ramas que dependen en gran medida de otras industrias. Parece razonable, sin 
embargo, seleccionar como industrias clave aquellas cuyos efectos se reflejan en muchas industrias porque sus efectos se difundirán en mayor medida en la red industrial.

Para medir la mayor o menor concentración de los efectos de arrastre se calculan los siguientes índices que recogen coeficientes de variación (desviación estándar con respecto a la media):

$$
v \cdot{ }_{j}=\frac{\left.\sqrt{\frac{1}{n-1} \sum_{i}\left(Z_{i j}-\frac{1}{n} \sum_{i}\right.} Z_{i j}\right)^{2}}{\frac{1}{n} \sum_{i} Z_{i j}}
$$

Que permite calcular si la industria $j$ arrastra o no uniformemente al sistema de industrias. Asimismo,

$$
v_{i} \cdot=\frac{\sqrt{\frac{1}{n-1} \sum_{j}\left(Z_{i j}-\frac{i}{n} \sum_{j} Z_{i j}\right)^{2}}}{\frac{1}{n} \sum_{j} Z_{i j}}
$$

Que permite calcular si el sistema de industria influye por igual (o no) sobre la rama $i$.

Pueden considerarse industrias clave aquellas con alto $U i$ (industrias con gran poder de dispersión de sus efectos sobre el sistema industrial y $V i$ relativamente bajo (la industria $i$ depende en gran medida, del sistema total y no de un escaso número de industrias). Es decir, las industrias clave tienen efectos importantes sobre muchas industrias. Véase esquema 2.

ESQUEMA 2

\begin{tabular}{|c|c|c|}
\hline$v_{\mathrm{i}}>1$ & $\begin{array}{l}\text { 1. Industrias clave } \\
\left(v_{i} \mathrm{y} v_{j} \text { pequeñas }\right)\end{array}$ & $\begin{array}{l}\text { 2. Industrias con efecto de } \\
\text { arrastre hacia adelante } \\
\left(v_{i} \text { pequeño }\right)\end{array}$ \\
\hline$v_{\mathrm{i}}<1$ & $\begin{array}{l}\text { 3. Industrias con efecto } \\
\text { de arrastre hacia atrás } \\
\left(v_{j} \text { pequeño }\right)\end{array}$ & $\begin{array}{l}\text { 4. Industrias } \\
\text { independientes }\end{array}$ \\
\hline
\end{tabular}

$$
v_{\mathrm{j}}>1 \quad v_{\mathrm{j}}<1
$$


Las industrias clave, así definidas, son importantes para el tratamiento de algunos problemas de política económica, porque son industrias que al ser impulsadas afectan a otras muchas y pueden dar lugar a un incremento generalizado de la actividad económica.

No obstante, Rasmussen señala que la consideración de una industria como clave sólo está en función del problema a tratar. El problema de política económica puede ser el aumento del empleo, la reducción de las importaciones o la eliminación de los estrangulamientos sectoriales. ${ }^{13}$

En algunos estudios (es decir, B.R. Hazari, 1970) se establece que para la consideración de industrias clave se debe tener en cuenta no sólo la capacidad de demanda de insumos intermedios de una industria, como en el caso anterior, sino también su papel en el sistema industrial en la medida en que sus productos pueden ser demandados como insumos intermedios por otras ramas. En este sentido, el papel de industrias clave queda más reforzado al ser éstas fuertemente demandantes y oferentes de insumos intermedios (además muy distribuidas tales demandas y ofertas por debajo de las $V i$ y $V j$ ). Esta definición de industria clave es similar a la definida por Hirschman, aunque éste no incorpora coeficientes de variación.

Los coeficientes de Rasmussen presentan algunas limitaciones.

a) Rasmussen previene contra la utilización simplista de estos coeficientes y más bien considera que el estudio de sectores clave es una forma abreviada de expresar las interdependencias que existen en una economía, así como un medio útil para considerar su evolución a lo largo del tiempo. En consecuencia, es fundamentalmente un elemento descriptivo y en este sentido tiene un gran valor para la comprensión de los procesos de desarrollo y cambio social.

b) Asimismo, se ha señalado su utilidad para expresar la coherencia de corto plazo de determinadas decisiones de política económica, así como su utilidad para el estudio de las industrias clave y la selección de los proyectos de inversión.

Actualmente, en los procesos de desarrollo, los temas relevantes a este respecto son, entre otros, el proceso de cambio tecnológico, el comercio interregional, etcétera.

13 Para una discusión sobre las variables económicas a considerar para una correcta identificación y selección de los sectores e industrias clave, a efectos del diseño de políticas regionales se puede consultar Oosterhaven (1981). 


\section{EVIDENCIA EMPÍRICA}

Para analizar empíricamente los sectores clave de la economía de BCS utilizamos la MIP estatal para 1993. ${ }^{14}$ La matriz de coeficientes técnicos es una matriz cuadrada de 72 sectores por 72 sectores. Sin embargo, a partir de ésta se obtuvo la matriz inversa de Leontief agregada solamente a 51 sectores por 51 sectores. $^{15}$

\section{Sectores clave Chenery-Watanabe}

Dicho esto pasamos a analizar los resultados empíricos concernientes a la identificación de los sectores clave de la economía sudbajacaliforniana, donde definimos a las industrias de acuerdo con sus encadenamientos hacia atrás y hacia delante, a la manera de Chenery y Watanabe.

La tipificación sectorial cuatripartita de Chenery-Watanabe establece:

1. Sectores base. De alto encadenamiento hacia adelante y bajo encadenamiento hacia atrás. Son sectores cuya demanda de insumos es pequeña, y cuya producción primaria es de destino intermedio; se inclina a abastecer de insumos a otros sectores, y canaliza una menor parte de producto al mercado como bien final (sectores de producción primaria intermedia).

2. Sectores clave. De alto encadenamiento hacia adelante y hacia atrás. Son fuertes demandantes de insumos intermedios, y fuertes oferentes de productos intermedios. Son sectores de paso obligado de los flujos sectoriales de la economía regional (sectores de manufactura intermedia).

3. Sectores con fuerte arrastre. De bajo encadenamiento hacia adelante y alto encadenamiento hacia atrás. Son sectores que muestran un consumo intermedio elevado, mientras que su oferta de productos irá principalmente hacia los consumidores finales (sectores de manufactura final).

4. Sectores independientes. De bajo encadenamiento hacia adelante y hacia atrás. Son sectores que consumen una cantidad poco significativa de insumos, y respecto a la distribución de su producción, la dedican principalmente a satisfacer la demanda final (sectores independientes).

Los resultados de esta clasificación sectorial se presentan en la siguiente gráfica:

14 N.A. Fuentes, A. Brugués, M. Ángeles y J. Sermeño, Matriz de insumo producto para Baja California Sur y sus encadenamientos intersectoriales, reporte técnico, SIMAC, 1998.

15 Existían ceros en algunas ramas y se decidió excluirlas para facilitar el manejo técnico de la MIP. 
GRÁFICA 1

CLASIFICACIÓN SECTORIAL

(indices de encadenamientos totales ponderados)

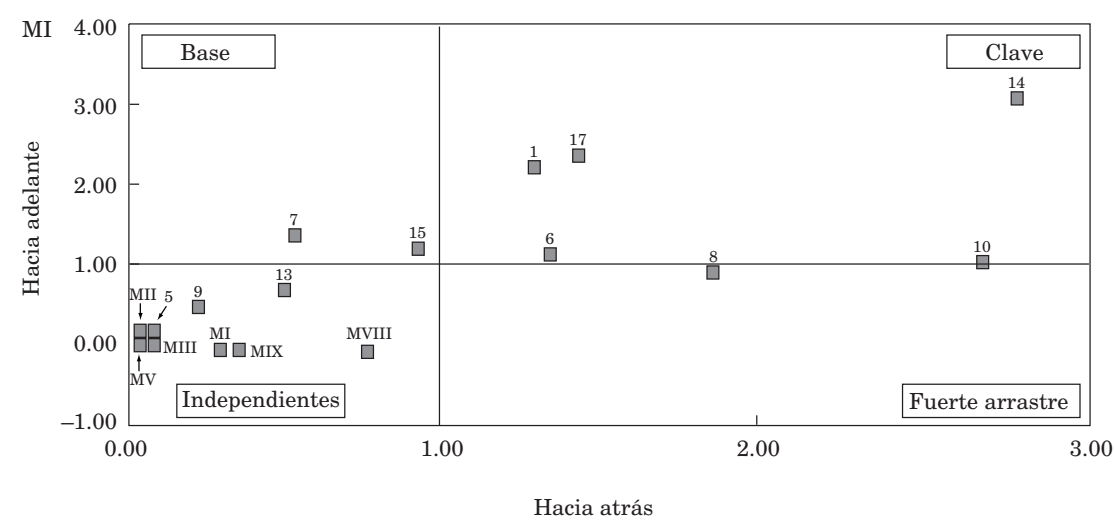

NOTA: La clasificación mostrada en la gráfica tiene una mayor desagregación de las ramas que la tabla.

En la gráfica 1 se puede observar que los sectores con mayores vinculaciones en la economía regional son: (14) Comercio, restaurantes y hoteles, (17) Servicios comunales, sociales y personales y (1) Agropecuario, silvicultura y pesca. Muestra también relaciones importantes a partir de los insumos que necesitan los sectores (10) Productos metálicos, maquinaria y equipo y Productos de minerales no metálicos. Exceptuando (8) Derivados del petróleo y carbón. ${ }^{16}$ Lo anterior pone en evidencia la importancia que los sectores relacionados con turismo y los del sector servicios del Estado tienen como potenciadores del crecimiento regional.

\section{Sectores clave Rasmussen}

Con respecto al análisis de los resultados empíricos concernientes a los sectores clave de la economía sudbajacaliforniana - si definimos a los sectores clave en términos puramente tecnológicos a la manera de Rasmussen y Hirschman - tenemos la tipificación sectorial cuatripartita siguiente:

1. Sectores clave. Pueden considerarse sectores clave aquellos en los que ambas $\mathrm{U}_{i}>1$ y $\mathrm{U}_{j}>1$ (industrias con gran poder de dispersión y sensibilidad de dis-

16 En la gráfica 1 se excluyeron los resultados para los setores de: (3) Productos Alimenticios, Bebidas y Tabaco y (16) Servicios Financieros, Seguros y Bienes Inmuebles, dado que presentaron valores positivos muy altos y por ello se perdía el detalle para el resto de los sectores. 
persión de sus efectos sobre el sistema industrial) y $\mathrm{V}_{i}$ y $\mathrm{V}_{j}$ relativamente bajos (las industrias $i$ y $j$ dependen en gran medida del sistema total y no de un escaso número de industrias). ${ }^{17}$

2. Industrias con efectos de arrastre hacia adelante. Pueden considerarse industrias con efectos de arrastre hacia adelante aquellas con $\mathrm{U}_{i}>1$ y relativamente bajo $V_{i}$.

3. Industrias con efectos de arrastre hacia atrás. Pueden considerarse industrias con efectos de arrastre hacia atrás aquellas con $U_{j}>1$ y relativamente bajo $V_{j}$.

4. Industrias independientes, $\mathrm{U}_{i} \mathrm{y} \mathrm{U}_{j}<1$.

Los resultados de esta clasificación sectorial se presentan en el cuadro 1:

CUADRO 1

SECTORES CLAVE BAJO EL CRITERIO DE ENCADENAMIENTOS (método de Rasmussen)

\begin{tabular}{rlcccc}
\hline & Sector & $U_{i}$ & $U_{j}$ & $V_{i}$ & $V_{j}$ \\
\hline 2 & Ganadería & 2.261 & 1.078 & 3.31 & 5.37 \\
18 & Alimentos para animales & 1.053 & 1.261 & 5.37 & 4.78 \\
19 & Otros productos alimenticios & 1.302 & 1.208 & 4.86 & 5.17 \\
45 & $\begin{array}{l}\text { Otros productos de minerales } \\
\text { no metálicos }\end{array}$ & 1.125 & 1.073 & 5.69 & 5.99 \\
\end{tabular}

FUENTE: Elaboración propia a partir de la MIP-BCS.

Como podemos notar, de acuerdo con este índice, los sectores que cuentan con ambos encadenamientos promedio mayores hacia adelante y hacia atrás y por tanto cumplen con los requisitos descritos en el inciso 1, son: (2) Ganadería; (18) Alimentos para animales, (19) Otros productos alimenticios, y (45) Otros productos de minerales no metálicos. Estos sectores se caracterizan porque ante un incremento en la demanda final de cualquier producto, sus requerimientos de insumos y su producción misma aumentan en promedio más que el resto de los sectores, por lo que incentivan la producción de otros sectores relacionados con ellos en el sistema. Por ejemplo, la rama (2) Ganadería, al aumentar su producción, estaría motivando un incremento directo del consumo intermedio de los sectores (1) Agricultura; (11) Productos cárnicos y

17 Bharat R. Hazari, "Empirical Identification of Key Sectors in the Indian Economy", The Review of Exonomics and Statistics 2, núm. 3, agosto de 1970. 
lácteos; (12) Envasado de frutas y legumbres; (13) Molienda de trigo y sus productos; (19) Otros productos alimenticios; (20) Bebidas alcohólicas; (24) Hilado y tejido de fibras blandas; (26) Otras industrias textiles; (28) Cuero y sus productos; (39) Jabones, detergentes, perfumes y cosméticos; (59) Otras industrias manufactureras; (69) Servicios de educación; (70) Servicios médicos; y (71) Servicios de esparcimiento. Estos sectores requieren de su producción para su propia actividad.

Por otro lado, (59) Otras industrias manufactureras, mediante sus encadenamientos directos hacia atrás, estaría motivando la producción de las ramas de quienes requieren insumos, a saber: (1) Agricultura; (2) Ganadería; (9) Canteras, arena, grava y arcilla; (11) Otros minerales no metálicos; (13) Molienda de trigo y sus productos; (18) Alimentos para animales; (19) Otros productos alimenticios; (26) Otras industrias textiles; (28) Cuero y sus productos; (30) Otras industrias de la madera; (31) Papel y cartón; (42) Artículos de plástico; (43) Vidrio y sus productos; (45) Otros productos minerales no metálicos, etcétera.

Los sectores que cuentan con mayores encadenamientos hacia atrás y un bajo coeficiente de variación se muestran en el cuadro 2. Este grupo de sectores es importante debido a la influencia que puede tener sobre la producción del resto de los sectores, ya que aun cuando no cuentan con un encadenamiento fuerte hacia adelante, se considera que estimulan la producción de insumos, por lo que su influencia en la economía debe ser tomada en consideración.

CUADRO 2

SECTORES CON ALTO ENCADENAMIENTO HACIA ATRÁS Y BAJO COEFICIENTE DE VARIACIÓN

(método de Rasmussen)

\begin{tabular}{rlrr}
\hline \multicolumn{1}{c}{ Sector } & $U_{j}$ & $V_{j}$ \\
\hline 8 & Minerales metálicos no ferrosos & 1.298 & 4.56 \\
11 & Productos cárnicos y lácteos & 1.596 & 4.37 \\
13 & Molienda de trigo y sus productos & 1.222 & 4.98 \\
14 & Molienda de nixtamal y productos de maíz & 1.177 & 5.55 \\
15 & Procesamiento de café & 1.120 & 5.26 \\
20 & Bebidas alcohólicas & 1.008 & 5.58 \\
22 & Refrescos embotellados & 1.168 & 4.94 \\
24 & Hilado y tejido de fibras blandas & 1.809 & 3.57 \\
28 & Cuero y sus productos & 1.228 & 4.89 \\
52 & Maquinaria y equipos eléctricos & 1.006 & 5.62 \\
58 & Otros equipos y material de transporte & 1.081 & 5.31 \\
60 & Construcción e instalaciones & 1.039 & 5.41 \\
67 & Alquiler de inmuebles & 1.039 & 5.47
\end{tabular}

FUENTE: Elaboración propia a partir de la MIP-BCS, 1993. 
El sector 24, Hilado y tejido de fibras blandas, destaca en este grupo. Como dijimos, este sector puede ser considerado de importancia por sus encadenamientos hacia atrás, pero si lo analizamos en un contexto más general, presenta una aportación negativa al valor agregado censal bruto, debido al alto valor que alcanza el rubro de otros conceptos del valor agregado censal bruto.

Esto puede contrarrestar el efecto positivo de la fuerza de sus encadenamientos hacia atrás y traducirse en una escasa aportación al crecimiento de la economía. Además, este mismo sector - (24) Hilado y tejido de fibras blandasutiliza poco menos del $45 \%$ de insumos regionales para su producción y el restante 55\% lo importa. Esta carga de importaciones puede tener efectos negativos en el nivel intersectorial, lo que induce a mayores importaciones si no se cuenta con una política que se oriente a satisfacer los requerimiento de insumos del sector, localmente.

Le sigue en importancia el sector (11) Productos cárnicos y lácteos. Este sector utiliza el $75.4 \%$ de insumos regionales para su producción, por lo que tiene sentido que cuente con altos encadenamientos intersectoriales hacia atrás. Por otro lado, en lo que respecta a su aportación al valor agregado, este sector tiene su mayor peso en el rubro de Remuneraciones.

A continuación, en el cuadro 3 se muestran aquellos sectores con mayores encadenamientos hacia adelante y con un bajo coeficiente de variación.

CUADRO 3

SECTORES CON ALTO ENCADENAMIENTO HACIA ADELANTE

Y BAJO COEFICIENTE DE VARIACIÓN

(método de Rasmussen)

\begin{tabular}{llcc}
\hline \multicolumn{1}{c}{ Sector } & $U_{i}$ & $V_{i}$ \\
\hline 66 & Servicios financieros & 1.150 & 4.93 \\
61 & Electricidad, gas y agua & 1.173 & 4.79 \\
62 & Comercio & 3.805 & 1.72 \\
64 & Transporte & 1.452 & 3.86 \\
65 & Comunicaciones & 1.195 & 4.81 \\
72 & Otros servicios & 1.186 & 4.79
\end{tabular}

FUENTE: Elaboración propia a partir de la MIP-BCS, 1993.

Clásicamente, estos sectores serán aquellos que ante un incremento en la demanda agregada, aumenten su producción de manera mayor al promedio, lo cual implicaría una oferta mayor en la economía para los demás sectores, y un estímulo para que éstos aumenten su propia producción. 
El sector que presenta un mayor encadenamiento hacia adelante es, como cabría esperar, el sector (62) Comercio. Lo que significa que ante un incremento en la demanda final de la economía, este sector incrementará sus requerimientos de productos de los demás sectores. Observado igualmente en un contexto más general, este sector mostró tener una importante participación en las importaciones, cuyo peso, de nuevo, se debe a la escasa relación que el sector guarda con los requerimientos de insumos industriales para su actividad. En este grupo es destacable la participación de los sectores de servicios, que conjuntamente con el sector Comercio, comunicaciones y transporte, permiten apreciar la importancia de los sectores terciarios en la economía.

\section{Sectores clave Hazari}

Con respecto al análisis de los resultados concernientes a los sectores clave de la economía sudbajacaliforniana - definidos éstos de acuerdo con una función de preferencia de los tomadores de decisiones de política (es decir, considerando la demanda final) y dada la estructura tecnológica-, en el cuadro 4 se muestran los sectores clave para la economía del estado. Los índices se construyeron utilizando una matriz con las demandas de cada uno de los sectores, y todos los demás mantienen su demanda en cero. De acuerdo con B. Hazari, esto nos estaría mostrando cuáles son aquellos sectores cuya demanda está ejerciendo mayor efecto sobre los demás, por lo que podemos identificarlos como estratégicos si consideramos que las preferencias de la gente están expresadas en la demanda final.

CUADRO 4

SECTORES CLAVE EN BAJA CALIFORNIA SUR

(método Hazari sin considerar ponderaciones)

\begin{tabular}{rlcc}
\hline \multicolumn{1}{c}{ Sector } & $Z_{i}$ & $Z_{j}$ \\
\hline 62 & Comercio & 791813.9 & 710244.6 \\
1 & Agricultura & 454830.6 & 412111.6 \\
19 & Otros productos alimenticios & 327192.0 & 402949.2 \\
63 & Restaurantes y hoteles & 349573.8 & 416012.4 \\
69 & Servicios de educación & 326788.2 & 349722.7 \\
10 & Otros minerales no metálicos & 263718.3 & 303172.4 \\
4 & Caza y pesca & 296091.8 & 334653.3 \\
\hline
\end{tabular}

FUENTE: Elaboración propia con base a la MIP-BCS, 1993.

El sector que aparece con un mayor índice dentro de todos es el 62, Comercio, en el cual tiene mayor importancia la demanda final de los productos que 
ofrece y un peso menor la de los insumos que requiere para su actividad. Por lo tanto, debe su importancia al factor de la demanda final de sus productos terminados, a saber: la demanda de los hogares, el consumo del gobierno, y las exportaciones. Lo mismo sucede en el caso del sector 1, Agricultura, en el cual tiene un mayor índice la demanda debida a los productos que ofrece el sector y no aquellos que requiere como insumos.

Una situación distinta se presenta en los sectores que le siguen en orden, puesto que en su caso, el mayor índice del sector esta representado por la demanda debida a los productos que utilizan como insumos. Éste es el caso de los sectores siguientes: (19) Otros productos alimenticios; (63) Restaurantes y hoteles; (69) Servicios de educación; (10) Otros minerales no metálicos; y (4) Caza y pesca.

Debido al criterio que originó la identificación de los sectores ubicados bajo el Método de Rasmussen, a saber, la demanda final de un sector, y conociendo que los sectores de demanda final en la matriz están conformados por: A) el consumo de los hogares, B) el consumo del gobierno, C) la formación bruta de capital, y D) las exportaciones, podemos intentar una clasificación de dichos sectores de acuerdo con el tipo de demanda final que los origina principalmente, por ejemplo:

CUADRO 5

SECTORES CLASIFICADOS DE ACUERDO CON EL TIPO DE DEMANDA FINAL QUE LOS ORIGINA

\begin{tabular}{rlc}
\hline \multicolumn{1}{c}{ Sector } & $\begin{array}{c}\text { Tipo de demanda } \\
\text { que lo origina* }\end{array}$ \\
\hline 62 & Comercio & A, B, C, D \\
1 & Agricultura & A, D \\
63 & Restaurantes y hoteles & A, B, D \\
69 & Servicios de educación & A, B \\
19 & Otros productos alimenticios & A, D \\
4 & Caza y pesca & A, D \\
10 & Otros minerales no metálicos & D \\
\hline
\end{tabular}

* Tipo de demanda: A: consumo de los hogares; B: consumo del gobierno;

C: Formación bruta de capital (FBKF) y D: exportaciones.

FUENTE: Elaboración propia con base en la información del cuadro 4.

Como podemos ver, los rubros que aparecen con mayor frecuencia son los de la demanda final proveniente del consumo de los hogares (A), y la proveniente de las exportaciones (D), que a la vez, como parte de la demanda final, representan alrededor del $72 \%$. En un segundo lugar estaría la demanda final del 
consumo del gobierno (B), que representa alrededor del 15\% de la demanda final. La demanda final proveniente de la formación bruta de capital o inversión podría ubicarse más adecuadamente si analizamos aquellos sectores que han sido beneficiados por inversiones privadas, o bien por algunos programas de gobierno; dentro de la demanda final, este rubro representa el de menor proporción (12.9 por ciento).

Si observamos el cuadro 4, el menor índice dentro de los sectores identificados lo presenta el sector 10 , Otros minerales no metálicos, el cual debe su demanda final en gran parte al rubro de exportaciones.

Ponderación de sectores para identificación de sectores clave Por último, los índices obtenidos en el cuadro 4 no están ponderados. Al ponderar por la demanda final, lo que estamos haciendo es estimar los efectos de primer orden en el producto debido a cambios en ésta. ${ }^{18}$

De acuerdo con la ponderación propuesta por el mismo B. Hazari, tenemos los resultados en el cuadro 6.

\section{CUADRO 6}

SECTORES PONDERADOS POR LA DEMANDA FINAL (método Hazari con ponderaciones)

\begin{tabular}{rlrr}
\hline \multicolumn{1}{c}{ Sector } & \multicolumn{1}{c}{$Z i W i$} & \multicolumn{1}{c}{$Z j W i$} \\
\hline 62 & Comercio & 133375.0 & 119635.3 \\
1 & Agricultura & 47325.0 & 42880.1 \\
63 & Restaurantes y hoteles & 32026.5 & 38113.3 \\
69 & Servicios de educación & 28893.7 & 30921.5 \\
19 & Otros productos alimenticios & 23180.4 & 28547.5 \\
4 & Caza y pesca & 21706.2 & 24533.1 \\
10 & Otros minerales no metálicos & 18668.9 & 21461.9
\end{tabular}

FUENTE: Elaboración propia con base en la MIP-BCS, 1993.

Los índices del cuadro 6 nos indican el peso que tiene cada sector de acuerdo con su importancia en la demanda final cuando los demás sectores se consideran con demanda cero. Como se puede apreciar, una vez ponderados los sectores por lo que respecta al impacto que tiene su demanda final en los demás sectores, los índices permanecen en los mismos lugares, en términos relativos a los

18 Zbigniew Kozikowski, Técnicas de planificación macroeconómica, México, Ed. Trillas, 1a. edición, mayo de 1988, p. 138. 
obtenidos mediante el método Hazari con ponderaciones. El único sector que gana cierta importancia es el sector 69, Servicios de educación, colocándose por encima del sector 19, Otros productos alimenticios. En términos absolutos, se puede decir que se confirma su posición como sectores estratégicos.

Ahora bien, comparando los sectores obtenidos bajo Rasmussen, con aquellos obtenidos bajo Hazari ponderado, sólo aparece un sector que es clave en ambos casos, a saber, el sector 19, Otros productos alimenticios.

Para tratar de entender a que se debe esta importancia, revisamos cómo está conformado el sector y encontramos los siguientes grupos y subgrupos de actividades: Grupo 190, Dulces, chocolates y confituras; Subgrupos 1901, Bombones, confituras, jaleas y dulces; 1902, Beneficio de cacao, cocoa y chocolate de mesa; 1903, Tratamiento y envasado de miel; 1904, Chicles; 1905, Flanes y gelatinas; y finalmente por el Grupo 191, Subgrupo 1911, Preparación y envasado de pescados y mariscos.

Por su importancia en la región, consideramos que el rubro referente al Subgrupo 1911, Preparación y envasado de pescados y mariscos, participa con mayor peso dentro de todas estas actividades. No es casual entonces que encontremos dentro de esta misma identificación de sectores clave obtenidos bajo el método Hazari sin ponderaciones, al sector 4, Caza y pesca, que suministra el insumo necesario para el anterior.

En este mismo orden de ideas, comparando los índices de Rasmussen y Hazari, podemos concluir que cuando se llevó a cabo una identificación de sectores bajo el criterio de sus encadenamientos con otros sectores en el sistema, encontramos que la importancia recayó principalmente en los sectores primario y secundario. Lo anterior, de acuerdo con Hazari, nos estaría indicando una relación estrictamente tecnológica, puesto que los sectores son identificados partiendo de sus relaciones interindustriales. En cambio, cuando la identificación involucra a la demanda final, aparecen en la escena con mayor peso los sectores terciarios, principalmente el 62, Comercio, seguido por los sectores primarios y por los de mayor peso en las exportaciones, principalmente 1 , Agricultura. Lo anterior nos puede indicar por lo menos dos cosas: primero, una economía con actividades predominantemente terciarias y con alta dependencia de las importaciones, puesto que la actividad comercial se realiza mayormente basada en éstas; y segundo, una economía con una base exportadora de productos primarios, principalmente.

\section{CONCLUSIONES}

El estado de Baja California Sur ha presentado durante las últimas dos décadas cambios importantes -eliminación del régimen preferencial de Zona 
Libre y reducción de subsidios federales -, lo cual se ha traducido en modificaciones tanto en su estructura económica como en el crecimiento de sus ciudades y población. Esto ha representado un enorme reto para la entidad, ya que debe hacer frente a las demandas de los diversos agentes económicos, lo que ha provocado una discusión de estrategias dirigidas a la búsqueda de un desarrollo económico estatal que le permita aprovechar sus potencialidades y desarrollar otras nuevas con una estrategia definida en el corto y largo plazos. La idea de encauzar a la región hacia un nuevo modelo de desarrollo resulta un tema de debate actual.

En los planes estatales de desarrollo 1987-1993, 1993-1999 y 1999-2005, así como en el proyecto Gran Visión se ha mencionado el concepto de sector clave y se ha apostado a la inversión en sectores específicos, pero en ninguno de los planes o proyectos de desarrollo se declara haber realizado una estimación de los impactos que esta inversión tendrá sobre el resto de los sectores, ni de otras variables relevantes como empleo, ingreso, valor agregado, importaciones y exportaciones. El Plan Estatal de Desarrollo vigente plantea la posibilidad de un nuevo modelo de desarrollo que contemple cuestiones distributivas, pero en el sentido estrictamente económico continúa sin ofrecer una alternativa que revierta la polarización actual de la estructura productiva.

Una preocupación que surge de esto es que los planes y programas de desarrollo no parten de bases sólidas en el sentido de proyectar y cuantificar las dimensiones y alcances en distintos sentidos - social, económico, ambiental- de las acciones que se pretenden realizar. Más aún, las estrategias elaboradas de esta forma caen en peligro de ser reactivas y no proactivas ante los problemas regionales. Los proyectos apoyados, sin tomar en cuenta las interrelaciones de la estructura productiva, terminan por obedecer a los beneficios directos que los mismos ofrecen de manera inmediata, y no a los impactos que indirectamente tienen en la economía, pensados desde una perspectiva de más largo plazo. Considerar lo anterior, contribuiría a cimentar una senda de desarrollo más sostenida.

Cuando se define un sector clave utilizando como criterio sus encadenamientos intersectoriales con otros sectores del sistema, y atendiendo al primer criterio establecido - índice de Chenery y Watanabe - encontramos que la importancia de BCS recae principalmente en los sectores siguientes:

62. Comercio

63. Restaurantes y hoteles

04. Caza y Pesca 
02. Ganadería

18. Alimentos para animales

11. Productos cárnicos y lácteos

19. Otros productos alimenticios

24. Hilado y tejido de fibras blandas

37. Resinas sintéticas y fibras artificiales

25. Hilado y tejido de fibras duras

35. Química básica

40. Otras industrias químicas

47. Industrias básicas de metales no ferrosos

45. Otros productos de minerales no metálicos (es decir, sal y yeso)

Estos sectores, de acuerdo con Chenery y Watanabe, corresponderían a los grupos de sectores con fuerte arrastre y sectores clave, en donde los primeros son fuertes demandantes de insumos intermedios, principalmente regionales, y por lo tanto tienen grandes posibilidades de arrastrar e inducir crecimiento económico, y los segundos son fuertes demandantes y oferentes de insumos intermedios, por lo que se dice son paso obligado de los flujos sectoriales de toda la economía.

En cuanto al segundo criterio -índice de Rasmussen incluyendo un bajo coeficiente de variación-, las estrategias del desarrollo tendrían que promocionar aquellos sectores que se consideran clave, según sus encadenamientos:

\section{Ganadería}

18. Alimentos para animales

19. Otros productos alimenticios

45. Otros productos de minerales no metálicos (es decir, sal y yeso)

Se observa que estos sectores son considerados clave por tener ambos tipos de encadenamiento - hacia adelante y hacia atrás - mayores a la media. Por lo tanto, presentan la mejor combinación de articulaciones con la economía de BCS.

Por otro lado, bajo el mismo criterio de encadenamientos, otros sectores de gran importancia por sus encadenamientos hacia atrás exclusivamente, son los siguientes:

08. Minerales metálicos no ferrosos

11. Productos cárnicos y lácteos

13. Molienda de trigo y sus productos 
14. Molienda de nixtamal y productos de maíz

15. Procesamiento de café

20. Bebidas alcohólicas

22. Refrescos embotellados

24. Hilado y tejido de fibras blandas

28. Cuero y sus productos

52. Maquinaria y equipos eléctricos

58. Otros equipos y material de transporte

60. Construcción e instalaciones

67. Alquiler de inmuebles

Estos sectores se encuentran muy articulados con la economía de BCS, por sus requerimientos de insumos regionales. El tamaño de algunos de ellos limita su potencial de impacto en la economía estatal.

En cuanto al tercer criterio -índice Hazari incluyendo ponderaciones-, se tiene que la estrategia de desarrollo busca promover a los sectores de acuerdo con su importancia en la demanda final, a saber:

62. Comercio

01. Agricultura

63. Restaurantes y hoteles

69. Servicios de educación

19. Otros productos alimenticios

04. Caza y pesca

45. Otros minerales no metálicos (es decir, sal y yeso)

Como se puede apreciar, una vez ponderados los sectores por lo que respecta al impacto que tiene su demanda final en los demás sectores, aparecen en la escena sectores muy distintos a los encontrados bajo el criterio de encadenamientos. Los sectores encontrados bajo el tercer criterio son los que tienen un fuerte dinamismo, pero una escasa interrelación con el resto de la economía, ya que sólo uno de ellos permanece con importancia cuando se consideran los encadenamientos, y éste es el sector 19, Otros productos alimenticios.

\section{Recomendaciones para el diseño de una estrategia de desarrollo}

Destacando los sectores identificados bajo el primero y segundo criterios - es decir, encadenamientos hacia atrás y hacia delante-, sería recomendable considerar dentro de la estrategia de desarrollo el fortalecer y potenciar algunos de 
estos sectores que resultaron ser clave, y otros que destacaron por sus encadenamientos hacia atrás, y que debido a su tamaño no representan un fuerte impacto en la economía regional; sin embargo, mantienen fuertes vínculos en la misma porque consumen insumos principalmente locales y debido a sus efectos multiplicadores. En general, partiendo de la hipótesis de una posible correlación entre encadenamientos $\rightarrow$ sectores clave $\rightarrow$ desarrollo económico, el apoyo que se brinde a los sectores identificados como clave y a los relacionados con los requerimientos de insumos de éstos, llevará a aumentar el tamaño de los primeros. Al ser el tamaño de los sectores mencionados parcialmente endógeno, pues depende del tamaño de otros sectores relacionados, existe la posibilidad de que al incentivarlos generen desarrollo económico para la región.

Por otro lado, y haciendo énfasis en los sectores clave identificados bajo el tercer método - es decir, Hazari ponderado-, encontramos que, a nuestro criterio, se deberían considerar dos clusters (grupos) económicos que son relevantes para el funcionamiento del sistema económico regional como sectores de apoyo, debido a su dinamismo. El primer cluster estaría constituido por actividades primarias: (01) Agricultura; (45) Productos minerales no metálicos (sal y yeso) y (04) Caza y pesca. El otro cluster relevante está constituido por actividades terciarias: (62) Comercio; (63) Restaurantes y hoteles. La conformación de estos clusters contribuiría a articular estas actividades económicas con el resto de la estructura económica, de tal forma que se generaran industrias o sectores que los utilizaran más intensamente en la economía de la entidad.

También, de acuerdo con los resultados, se observa que en el nivel estatal existe una significativa proporción de sectores que presentan una relativa independencia con respecto al resto de los otros sectores, lo que nos habla de una economía con una estructura productiva escasamente articulada. En este sentido, consideramos que una estrategia de desarrollo que se encamine a articular el sistema productivo, puede representar una alternativa de más largo plazo.

Finalmente, debemos destacar que este análisis tiene sus limitaciones derivadas de la estimación de la MIP para el estado, y de los índices utilizados. Sin embargo, consideramos que los resultados obtenidos en este estudio aportan algunos elementos para la realización de otros estudios y para el análisis de las características de la economía de BCS y de su proyecto de desarrollo económico estatal.

\section{BIBLIOGRAFÍA}

- Andersen Arthur \& Co. S.C. Gobierno del Estado de Baja California Sur (1992), Programa Estratégico de Desarrollo Económico para el Estado de B.C.S., 4 vols., La Paz, B.C.S., México. 
- Baca Tupayachi, Epifanio, Ruth García Pacheco, Leonith Hinojosa Valencia, Jesús Guillén Marroquín y Guillermo Mosqueira Lovón (1993), Análisis de la economía de la región inka con base en las tablas de insumo producto, Cuzco, Perú, Centro de Estudios Regionales Andinos Bartolomé de Las Casas.

- Chenery, H.B. (1961), "Comparative Advantage and Development Policy", en American Economic Review, vol. 51, marzo, pp. 18-51.

- y y T. Watanabe (1958), "An International Comparison of the Structure of Production", Econométrica, vol. 26, 4 de octubre, pp. 487-521.

- Fuentes, N.A., A. Brugués, M. Ángeles, y J. Sermeño (1998), "Matriz de insumo producto para Baja California Sur y sus encadenamientos intersectoriales", reporte técnico, La Paz, SIMAC.

- Garza, Mónica de la (1988) (compiladora), Eslabonamientos productivos en Argentina, Brasil y México (II Seminario Internacional), México, Universidad Autónoma Metropolitana, División de Ciencias Sociales y Humanidades.

- Garza, Mónica de la y Aníbal Zottele Allende (1986) (coordinadores), Bloques de interdependencia: mercado de trabajo y estudios de caso, México, Universidad Autónoma Metropolitana, División de Ciencias Sociales y Humanidades.

- Hirschman A.O. (1958), The Strategy of Economic Development, New Haven.

- Hazari, B.R. (1976), "Empirical Identification of Key Sectors in the Indian Economy", en The Review of Economics and Statistics, vol. xx, pp. 301-305.

- Harrison, B. (1992), "Industrial Districts: Old Wine in New Bottles?", en Regional Studies, vol. 26, núm. 5, pp. 469-483.

- Hewings, Goeffrey J.D. (1982), "The Empirical Identification of Key Sector in a Economy: a Regional Perspective", en The Developing Economies, vol. 2, núm. 20.

- Leontief, Wassily (1936), "Quantitative Input and Output Relations in the Economic System of the United States”, en The Review of Economic Statistics, vol. xvIII, núm. 3. Reproducida en Heinz D. Kurz, Erik Dietzenbacher y Christian Lager (1998), Input-Output Analysis, vol. I, Edward Elgar Pub.

- Lifschitz, Edgardo y Aníbal Zottele Allende (1985) (coordinadores), Eslabonamientos productivos y mercados oligopólicos, México, Universidad Autónoma Metropolitana, División de Ciencias Sociales y Humanidades.

- Oosterhaven, Jan (1981), Interregional Input-Output Analysis and Dutch Regional Policy Problems, University of Groningen, The Netherlands, Gower Publishing Company Limited, Hampshire, Inglaterra, 1981.

- Plan Estatal de Desarrollo 1987-1993, Baja California Sur. 
- Plan Estatal de Desarrollo 1993-1999, Parte II, "Estrategia de Desarrollo", Baja California Sur.

- Plan Estatal de Desarrollo 1999-2005, Baja California Sur.

- Porter, M. (1998), "Clusters and the New Economics of Competition", en Harvard Business Review, noviembre-diciembre.

- Rasmussen, P.N. (1956), Studies in Inter-sectorial Relations, Copenhague.

- Schultz, S. (1976), "Intersectorial Comparison as an Approach to the Identification of Key Sectors", en Polenske and Skolka, Advances in Input-output Analysis, Cambridge, Mass. 